Monostori, L.; Kádár, B.; Viharos, Zs.J.; Stefán, P.; AI and machine

learning techniques combined with simulation for designing and

controlling manufacturing processes and systems, Preprints of the IFAC

Symposium on Manufacturing, Modeling, Management and Supervision,

MIM 2000, July 12-14, 2000, Patras, Greece, pp. 167-172.

\title{
AI AND ML TECHNIQUES COMBINED WITH SIMULATION FOR DESIGNING AND CONTROLLING MANUFACTURING PROCESSES AND SYSTEMS
}

\author{
László Monostori, Botond Kádár, Zsolt Viharos, István Mezgár, Péter Stefán
}

Computer and Automation Research Inst., Hungarian Academy of Sciences

Kende u. 13-17, H-1518 Budapest, POB 63, Hungary

\begin{abstract}
In the paper different architectures with partly self-developed simulation packages are described illustrating the benefits of combining simulation and machine learning (ML) techniques in manufacturingintelligence (AI) and ML side, artificial neural networks, heuristic search, simulated annealing, and agent-based techniques are put into action. The applicability of the proposed solutions is illustrated by the results of experimental runs. Copyright (C) 1999 IFAC
\end{abstract}

Keywords: Production, Simulation, Artificial intelligence, Machine learning

\section{INTRODUCTION}

Simulation is usually an efficient technique to make difficult problems more tractable. It can contribute to elaborating new algorithms, supporting decision makers, decreasing the risk in investments, and running the systems exposed to changes and disturbances more efficiently.

From simulation point of view, one can speak about knowledge-based hybrid systems (KBHSs) if simulation and some kind of intelligent techniques, e.g. expert systems (ESs), artificial neural networks (ANNs), fuzzy systems or their combination are used together. Without aiming at completeness, four main architectural categories of this special group of hybrid systems can be distinguished (Fig. 1):

- $\quad$ Embedded: The simulation model is embedded in a KBS, which can use simulation (SIM) in decision making (A), or the simulation model contains a KBS (B), (e.g. for simulating uncertain processes).

- Parallel: The simulation communicates with the user and can access the results of the decisions made by the KBS (C), or the KBS communicates with the user, and controls the simulation (D), which can be replaced later by the real system.

- Co-operative: The KBS contains some knowledge about the simulation model as well as about the field of application (E). This form of combination is very useful for users nonexperienced in simulation, by helping them building or modifying the model.

- Intelligent front-end: The KBSs generate the necessary instructions for the user from different aspects, interpret and explain the results from the simulation package (F).

Learning denotes changes in the system that is adaptive in the sense that they enable the system to do the same or similar task more effectively next time (Simon, 1983). Obviously, ML techniques can enhance the performance of any KBHS architecture of Fig. 1, and at the same time, simulation can be used for generating training examples for learning. 

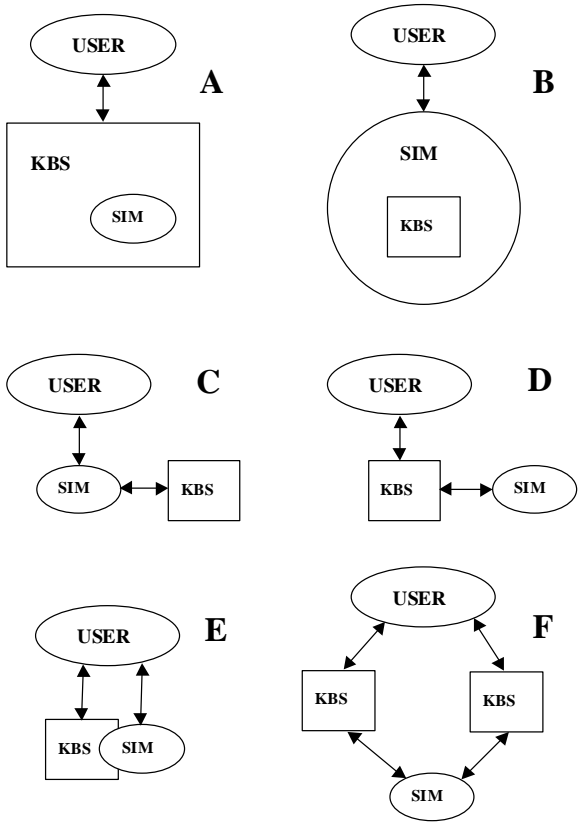

Fig. 1. Possible combinations of simulation and KBSs

The paper illustrates the benefits of combining simulation and machine learning techniques in three fields:

- modelling, simulation and optimisation of production processes and process chains,

- design, control and reconfiguration of flexible manufacturing systems (FMSs),

- design and control of holonic manufacturing systems (HMSs).

\section{SIMULATION AND OPTIMIZATION OF PRODUCTION PROCESSES AND PROCESS CHAINS}

Difficulties in modelling production processes are manifold: the great number of different machining operations, multidimensional, non-linear, stochastic nature of machining, partially understood relations between parameters, lack of reliable data, etc. A number of reasons back the required models: design, optimisation, control and simulation of processes and design of equipment (Van Luttervelt, et al., 1998, Merchant, 1998).

An approach based on back propagation ANNlearning and heuristic search for generating multipurpose models of production processes which are applicable for a set of assignments and can satisfy the various accuracy requirements was described in (Monostori and Viharos, 1999). As to the application phase, a novel technique based on simulated annealing search was developed to find the unknown parameters of the model in certain situations.

In the following space a block-oriented software named 'ProcessManager' for optimising operations and/or production chains form various points of view at the same time, will be introduced. The simulation technique is based on multipurpose ANN-models trained by using measured data. Multiple objectives are handled with the usual weighting technique.
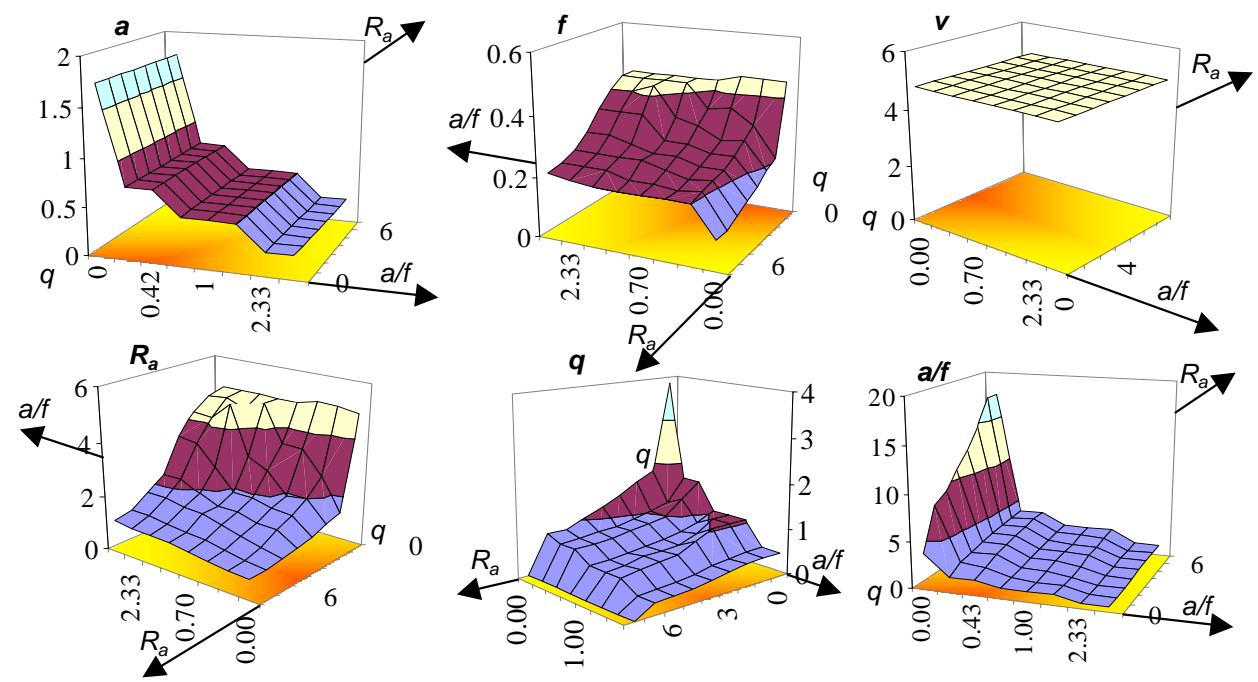

Fig. 2. Parameters resulted by the threefold optimisation of the plate turning operation (normalised values)

Fig. 2 illustrates the application of ProcessManager for the threefold optimisation of the viewpoints of the customer (minimisation of the surface roughness, $R_{a}$ ), owner of the company $(q$, profit/productivity maximisation) and the production engineer (maximisation of process stability through the $a / f$ ratio). Parameters resulted from the optimisation of the plate turning operation are illustrated by 3D-plots. 
Ratios of the weighting factors of the three variables to be optimised are represented along the axes. The 'surfaces' are to be used together, i.e. the movement on the plane marked by $R_{a}$ and $a / f$ occurs on each of the diagrams at the same time. The corner marked by $q$ indicates the position where the viewpoint of the company owner is the most important and the movement along the axes $R_{a}$ and $a / f$ represents that the corresponding criteria become more and more important with respect to $q$. The results can be directly used for supporting business decisions and compromises.

\subsection{Simulation and optimisation of process chains}

The sequence of production operations can be modelled by a chain of models connected by their input-output parameters (Westkämper, 1995). In addition to process optimisation, ProcessManager supports the modelling and optimisation of process chains as well.

ProcessManager incorporates (Fig. 3. ):

- Definition of the elements of the chain..

- Determination of the process models in a hybrid way, by integrating analytical equations, expert knowledge and example-based learning.

- Connection of the single models into a process chain by coupling input-output model parameters not limited to models of successive processes in the chain.

- Definition of eligible intervals or limits for the process parameters and monitoring indices.

- Definition of a cost function to be optimised, etc.

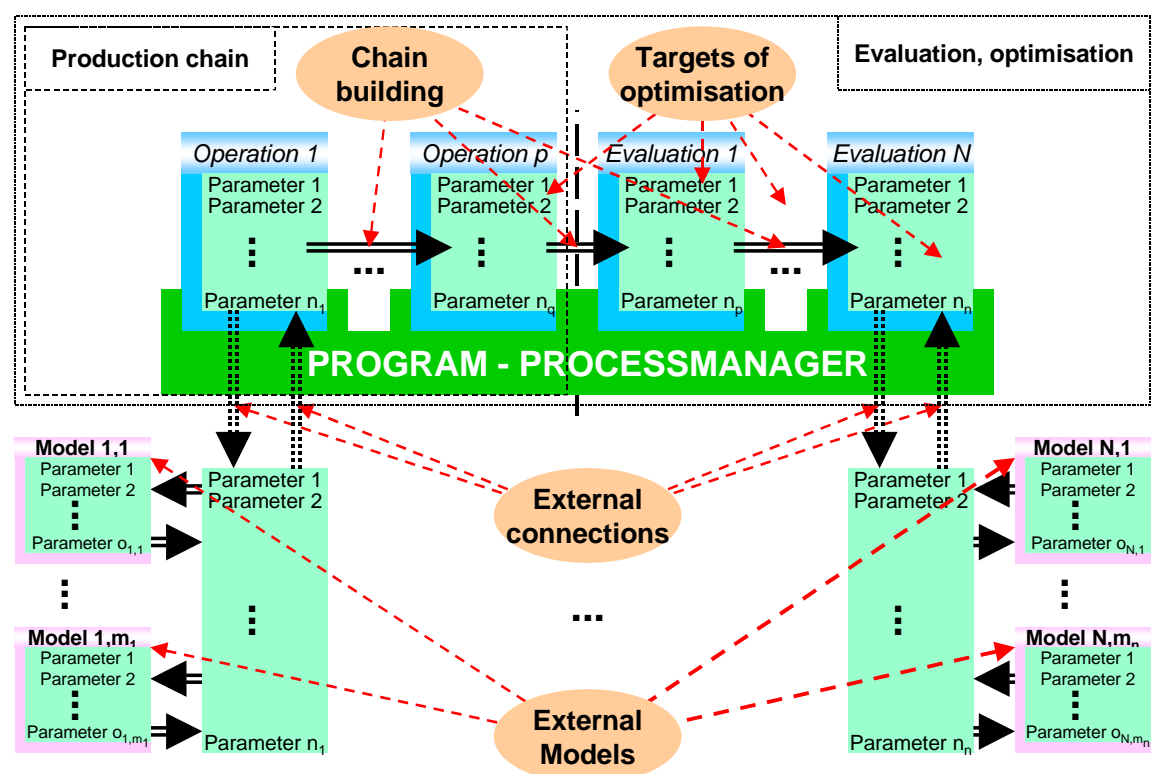

Fig. 3. Hybrid modelling and optimisation of process chains by ProcessManager

\section{DESIGN, CONTROL AND RECONFIGURATION OF FMSs}

An FMS design methodology which combines design of experiments (DoE) technology, Taguchi method, and knowledge based simulation techniques was described in (Mezgár et al., 1997). The design of new FMSs is not a daily assignment, but their redesign, reconfiguration for a new product, or in case of different disturbances is a very frequent task. The application of simulation techniques is usually time consuming, which is tolerable in the design phase, but it is hardly acceptable in real manufacturing situations. As a reasonable solution, the substitution of the simulator by ANNs for mapping between design factors and system performance is proposed. The applicability of the approach is demonstrated by

- the estimation of the throughput time of FMSs,

- $\quad$ and the determination of the appropriate speed of the AGV in the analysed system.

\subsection{Simulation and ANN-based learning and estimation of the throughput time}

During the described investigations three-layer back propagation (BP) neural networks were applied for the FMS at the TU Budapest (Mezgár et al., 1997). Sufficient number of pattern-target pairs were generated by simulation to cover an appropriate broad combination of design, indicative and noise 
factors. The number of machine tools and the place of measurement were considered as design factors. The speed of the robots, and the AGV have been defined as indicative factors. The number of scrap in a batch, the machine set-up and maintenance time, furthermore, the frequency and duration of toolchange were selected as noise factors.

The networks were set up as shown in Fig. 4. Design, indicative and noise factors constituted the 9 elements of input patterns for three-layer networks with the variable number of hidden neurons (9-X-1 structure). During learning 104 simulated values were used as targets. The best estimation results for test patterns were reached by the network of 9-5-1 structure (7,3\% maximum, and 3,2\% average relative error). These results projected success in applying neural networks trained by simulation results for throughput time estimation.

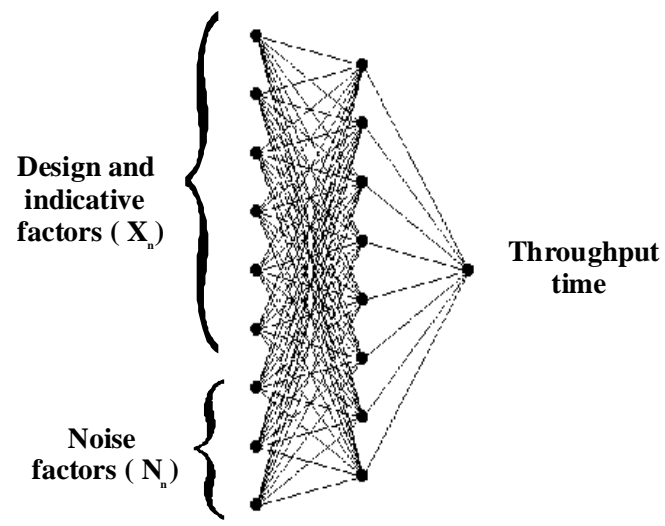

Fig. 4. Artificial neural network for throughput time estimation

\subsection{Combined use of DoE and ANN techniques in the reconfiguration of manufacturing systems}

The goal in the reconfiguration phase is to develop a new version of the basic configuration, while maintaining the original basic characteristics of the system as much as possible. Motivations of reconfiguration are manifold: introduction of a new product within the family, machine brake-down, the modification of the process plan or the deadline of shipping, etc.

Reconfiguration usually leads to an iterative simulation-evaluation procedure. Generation of a network which, in some sense, realises the inverse of the simulation function is of significant importance (Chryssolouris et al., 1987). Fig. 5 illustrates a simplified problem setting. The speed of the AGV in the system is to be determined on the base of the other (design, indicative and noise) factors kept constant, and the required throughput time of the system.

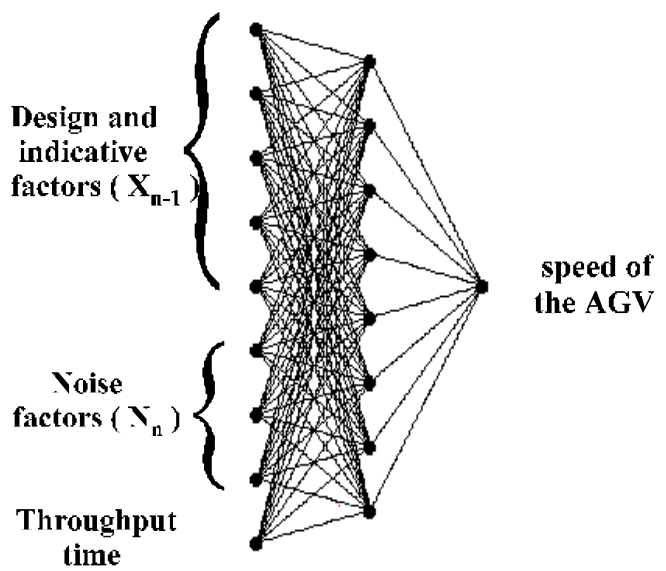

Fig. 5. Artificial neural network for determining the speed of the AGV in the reconfiguration process

Through the appropriate use of the training patterns the weights of the 9-8-1 network in Fig. 5 were generated by BP learning. The estimation of the AGV speed for test pattern resulted in a 5,2\% maximum, and a 2,1\% average relative error.

The result indicates the applicability of this procedure (i.e. realisation, in some sense, of the inverse of the simulation function by ANNs) for reconfiguring manufacturing systems, substituting the highly iterative, time consuming process.

\section{DESIGN AND CONTROL OF HMSs}

Management of complexity, changes and disturbances is one of the key issues in production today (Wiendahl and Scholtissek, 1994). Holonic manufacturing systems (HMSs), as one of the new paradigms in manufacturing, consist of autonomous, intelligent, flexible, distributed, co-operative agents or holons (Van Brussel et al., 1996). They represent viable alternatives to hierarchical and heterarchical structures and the corresponding reactive scheduling approaches. The industrial acceptance of holonics, however, is relatively low among others things by reasons of

- $\quad$ the relative crudeness of the agent theory and its manufacturing applications,

- the insufficient communication and decision making capabilities of present NCs,

- $\quad$ the high investment costs of a production system working according to the agent principles,

- the seemingly insurmountable difficulties in their stepwise integration into existing production systems (Kádár and Monostori, 1998).

Several approaches ere introduced and treated in (Kádár and Monostori, 1998). to overcome the above difficulties: 
- $\quad$ the use of simulation technique for developing agent-based control architectures,

- the holonification of existing resources,

- $\quad$ the holonification of traditional systems by using the virtual manufacturing (VM) concept.

Here, we concentrate on the first and the third approach where simulation is a key issue.

\subsection{Development of agent-based architectures by simulation}

There are a number of open questions in holonics (Kádár et al., 1997) which can be answered by extensive simulation only. The object-oriented simulation framework for the development and evaluation of distributed manufacturing architectures described in (Kádár et al., 1997) provides a root model that represents a plant and can contain different agents. The object library incorporates two main agent types: resource agent and order agent. A plant in the model will contain only one order agent which is responsible for order processing, job announcements and job dispatching between different resources or groups (Fig. 6).

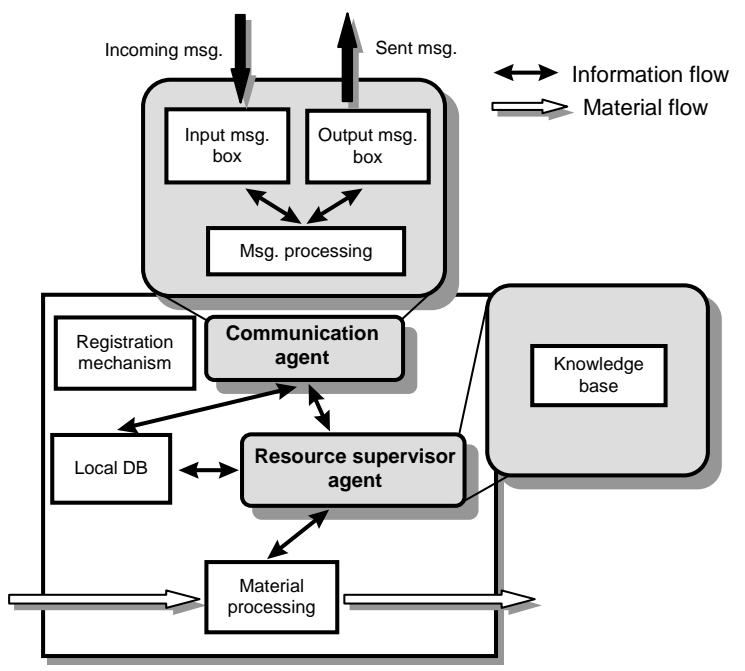

Fig. 6. Structure of a resource agent (Kádár et al., 1997)

A model may incorporate several resource objects which can be initialised during construction (giving the name of the resource, process-capabilities of the resource, etc.). Only one information provider, i.e. the registration book, is treated centrally in the system.

The simulation framework is intensively used for research purposes. A new approach to agent-based scheduling developed and tested by the framework is described in (Monostori et al. 1998).

\subsection{Holonic control of traditional systems by using simulation}

In this Section a novel approach to holonification of whole manufacturing systems is introduced based on an extension of the Virtual Manufacturing (VM) concept (Onosato and Iwata, 1993). Manufacturing sub-systems can be classified into four categories: Real Physical System (RPS), Real Informational System (RIS), Virtual Physical System (VPS), Virtual Informational System (VIS). VM makes it possible to simulate manufacturing processes in advance, without using real facilities, and by this way to accelerate the design and re-design of real manufacturing systems.

A fundamental feature of the VM concept is that it realises a one-to-one mapping between the real and virtual systems, i.e. VIS and VPS try to simulate RIS and RPS, respectively, as exactly as possible. In this section an extension of VM concept is suggested and illustrated. The main novelty of the approach is the break with the above one-to-one mapping, more exactly the use of the VM concept to control a traditional (centralised / hierarchical) manufacturing system in a holonic way.

Supposing that there is a central control unit in the traditional system, the fundamental requirements for the holonification of this system by the approach suggested here are as follows. The capabilities to communicate with the outside world, transfer control information to the resources, catch state information and to transfer them to the central unit, interrupt the functioning of the resources at given periods, stop or modify the processes started previously.

The virtual part of the system runs in a holonic way and incorporates order management, scheduling and control issues. For the realisation of the virtual part, simulation systems such as the framework described earlier in this Section can be advantageously used. Resource agents which, from technological point of view, correspond to the real resources of the traditional system can be easily constructed by using the object library of the simulation framework (Kádár et al., 1997). Order management proceeds fully in the virtual system.

Decisions are made in the virtual, holonic system and conveyed to the VIS of the traditional system. The real production situation is sensed by the RPS and forwarded to the VIS, which initiates appropriate measures in a holonic way. As a summary, the traditional system shows a holonic behaviour.

The holonic information system tested in a virtual environment has the potential of being used in real holonic systems. 


\section{CONCLUSIONS}

Some examples were described in the paper for applying simulation and $\mathrm{AI} / \mathrm{ML}$ techniques for different fields of manufacturing:

(a) modelling, simulation and optimisation of production processes and process chains,

(b) design, control and reconfiguration of flexible manufacturing systems (FMSs),

(c) design and control of holonic manufacturing systems (HMSs).

In (a) simulation incorporated trainable process models realised by ANNs was introduced. In respect to the notions of Figure 1, this integration approach belongs to category $\mathrm{E}$, however, depending on the way of realisation, it can belong to category B or C. In (b) the usual simulation technique was substituted by an ANN trained by the simulator. This approach can be ordered to category $\mathrm{D}$.

The case (c) addressed the application of simulation for the design and control of HMSs. The later approach was especially interesting: agent based control of centrally or hierarchically structured systems by using simulation techniques was proposed. The solution can be put in category B (or $\mathrm{C}$ or $\mathrm{E}$, depending on the given realisation/application).

Taking, on the one hand, the availability of the rapidly growing computing power, and on the other hand, the new achievements in AI/ML (e.g. reinforcement learning), into account, a versatile and rapidly increasing application of this type of KBHSs is expected in nearly every field of manufacturing.

\section{ACKNOWLEDGEMENTS}

This work was partially supported by the National Research Foundation, Hungary, Grant Nos. F026326 and T026486. A part of the work was covered by the Nat. Comm. for Techn. Dev., Hungary Grants (EU96-B4-025 and EU-97-A3-099) promoting Hungarian research activity related to the ESPRIT LTR Working Groups (IiMB 21108 and IMS 21995).

\section{REFERENCES}

Chryssolouris, G., M. Guillot and M. Domroese (1987). An approach to intelligent machining, Proc. of the 1987 American Control Conf., June 10-12, Minneapolis, MN, pp. 152-160.

Kádár, M. and L. Monostori (1998). Agent based control of novel and traditional production systems, Proc. ICME98, CIRP Int. Seminar on Intelligent Computation in Manuf. Eng., July 1-3, Capri, Italy, pp. 31 - 38. (key-note paper)
Kádár, B., L. Monostori,. and E. Szelke (1997). An object oriented framework for developing distributed manufacturing architectures, Proc. of the Second World Congress on Intelligent Manufacturing Processes and Systems, June 1013, Budapest, Hungary, pp. 548-554., and in Journal of Intelligent Manufacturing, 9, No. 2, April, 1998, pp. 173-179.

Merchant, M.E. (1998). An interpretive look at $20^{\text {th }}$ century research on modelling of machining, Inaugural Address, Proc. of the CIRP International Workshop on Modelling of Machining Operations, May 19, Atlanta, Georgia, USA, pp. 27-31.

Mezgár, I., Cs. Egresits and L. Monostori (1997). Design and real-time reconfiguration of robust manufacturing systems by using design of experiments and artificial neural networks, Computers in Industry, 33, No. 1, pp. 61-70.

Monostori, L., A. Márkus, H. Van Brussel and E. Westkämper (1996). Machine learning approaches to manufacturing, CIRP Annals, 45, No. 2, pp. 675-712.

Monostori, L., B. Kádár and J. Hornyák (1998). Approaches to manage changes and uncertainties in manufacturing, CIRP Annals, 47, No. 1, pp. 365-368.

Monostori, L. and Zs.J. Viharos (1999). Multipurpose modelling and optimisation of production processes and process chains by combining machine learning and search techniques, Proc. of The $32^{\text {nd }}$ CIRP Int. Sem. on Manufacturing Systems, May 24-26, Leuven, Belgium, pp. 399-408.

Onosato, M., and K. Iwata (1993). Development of a virtual manufacturing system by integrating product models and factory models, CIRP Annals, 42, No. 1, pp. 475-478.

Simon, H. A. (1983). Why should machines learn? In: Machine Learning: An Artificial Intelligence Approach, Tioga Press, pp. 25-38.

Van Brussel, H., P. Valckenaers, J. Wyns, L. Bongaerts, and J. Detand (1996). Holonic manufacturing systems and IiM. In: IT and Manufacturing Partnerships, Conf. On Integr. in Manufact., Galway, Ireland, pp. 185-196.

Van Luttervelt, C.A., T.H.C. Childs, I.S. Jawahir, F. Klocke and P.K. Venuvinod (1998). Present situation and future trends in modelling of machining operations, CIRP Annals, 47, No. 2, pp. 587-626.

Westkämper, E. (1995). Supervision of quality in process chains by means of learning process models, Proc. of the Second Int. Workshop on Learning in IMSs, April 20-21, Budapest, Hungary, pp. 566-590.

Wiendahl, H.-P. and P. Scholtissek (1994). Management and control of complexity in manufacturing. CIRP Annals, 43, No. 2, pp. 533540. 